\title{
LARGE SCALE VARIATIONS IN SOLAR CORONAL DENSITY AND MAGNETIC FIELDS
}

\section{By}

\section{R. M. McQueen}

High Altitude Observatory, Boulder, Colorado 80307,U.S.A.

Observations of the solar equatorial electron scattered coronal radiance made during the Skylab mission show characteristics which dramatically differ with time. The results may be interpreted as indicating a general simplification of the coronal magnetic field, and, in comparison with harmonic analysis of observed solar surface magnetic fields, as indicating a rapid response of outer coronal structures to abrupt changes in the global solar surface field structure. 